\title{
Salvianolic acid B prevents steroid-induced osteonecrosis of the femoral head via PPAR $\gamma$ expression in rats
}

\author{
SHUANGQING LI ${ }^{1}$ and JUAN WANG ${ }^{2}$ \\ ${ }^{1}$ Department of Orthopedics, Cangzhou Central Hospital of Hebei; ${ }^{2}$ Department of Educational Administration, \\ Cangzhou Medical College in Hebei, Cangzhou, Hebei 061001, P.R. China
}

Received August 27, 2015; Accepted October 5, 2016

DOI: $10.3892 /$ etm.2016.4008

\begin{abstract}
Salvianolic acid B (Sal B) is a water-soluble phenolic compound derived from Salvia Miltiorrhiza. Recent studies show Sal B has a clear function of anti-cerebral ischemia injury, which is closely related to antioxidation, free radical scavenging, neuroprotection and the blood brain barrier. The aim of the present study was to verify whether Sal B prevents steroid-induced osteonecrosis of the femoral head and to investigate its underlying pharmacological mechanisms. Steroid-induced osteonecrosis rat models were established to evaluate the effects of Sal B on osteonecrotic changes and repair processes. The use of Sal B improved steroid-induced histopathological scores and inhibited osteoclast differentiation in rats. Notably, Sal B induced bone marrow-derived mesenchymal stem cells into osteogenesis. Moreover, Sal B treatment suppressed peroxisome proliferator-activated receptor (PPAR) $\gamma$ and AP2 protein expression levels and increased runt-related transcription factor 2 and Collagen I protein expression levels in steroid-induced rats. osteocalcin and alkaline phosphatase content in steroid-induced rats was enhanced by treatment with Sal B. These results suggest that Sal B prevents steroid-induced osteonecrosis of the femoral head via PPAR $\gamma$ expression in rats. The present pilot study provides a brief insight into the effect of Sal B on steroid-induced osteonecrosis.
\end{abstract}

\section{Introduction}

Avascular necrosis is one of the most common clinical refractory diseases. According to reports, there are 50-75,000 new cases of avascular necrosis of the femoral head (ANFH) in China (1). Steroid-induced necrosis of the femoral head (SANFH) is the most common cause of non-traumatic femoral

Correspondence to: Mr. Shuangqing Li, Department of Orthopedics, Cangzhou Central Hospital of Hebei, 201 Xinhua Road, Cangzhou, Hebei 061001, P.R. China

E-mail: lianzikan5137@126.com

Key words: salvianolic acid B, steroid-induced osteonecrosis, femoral head, peroxisome proliferator-activated receptor $\gamma$ head necrosis (2). With the application of hormone therapies for immune system diseases, spinal cord injury and other diseases, there is an increasing trend in the incidence of SANFH (3). SANFH is most common in young adults, is often bilateral with high morbidity, and if left untreated $70 \%$ of the femoral head will collapse, which may result in serious mental stress and financial burden to individuals and society (4).

In SANFH, the bone cycle is interrupted, the active ingredient of bone is dead and a series of complex pathological processes can be observed, such as bone repair (5). The specific pathogenesis remains unclear, and possible mechanisms include endothelial injury, abnormal coagulation and fibrinolysis, lipid metabolism, bone metabolism and hemodynamic changes, which ultimately cause blood clots in the femoral head, the accumulation of fat cells, increased pressure in the bone, bone microcirculation disorders and osteonecrosis (6).

Osteonecrosis treatment includes non-surgical treatment and surgical treatment. The former includes weight reduction, medication, pulsed electromagnetic fields, high pressure oxygen and extracorporeal shock wave treatment; whereas the latter includes core decompression, bone graft without/with vascular intervention, osteotomy surgery, reconstruction of femoral head with support frame and hip arthroplasty (7). The selection of treatment method predominantly depends on the stage of osteonecrosis and the pathological range.

Salvianolic acid B (Sal B) is a water-soluble phenolic compound isolated from Salvia Miltiorrhiza, of which the active ingredient in water-soluble phenolic compound is $>50 \%$ (8). It has previously been shown that Sal B may significantly improve neuronal injury induced by acute cerebral ischemia and reperfusion, and improve the behavioral disorders caused by cerebral ischemia (9). It has also been demonstrated that Sal B has antioxidation, anti-amyloid aggregation, and anti-amyloid cytotoxic effects (10-12). In addition, Sal B is able to significantly promote the proliferation of vascular endothelial cells, playing the role of mitogen (13). The present study aimed to verify whether Sal B prevents SANFH via peroxisome proliferator-activated receptor (PPAR) $\gamma$ expression in rats.

\section{Materials and methods}

Animals. A total of 50 male Wistar rats (age, 12 weeks; weight, 300-320 g) were obtained from the Experimental Animal 
Centre of Cangzhou Central Hospital of Hebei province, and maintained in a room equipped with an air-filtering system, the cages and water were sterilized, and $23-24^{\circ} \mathrm{C}$. The present study was conducted at the Experimental Animals Research Laboratory of the Cangzhou Central Hospital of Hebei province (Cangzhou, China) according to The Guide for the Care and Use of Laboratory Animals. Approval was granted by the ethical commitee of this institution.

Groups and treatment. All rats were weighed and randomly divided into three groups after one week of feeding adaptation: Control group $(n=10)$; model group $(n=20)$; and Sal B group $(40 \mathrm{mg} / \mathrm{kg} / \mathrm{d} \mathrm{Sal} \mathrm{B} ; \mathrm{n}=20)$. A total of $21 \mathrm{mg} / \mathrm{kg}$ methylprednisolone acetate (Sigma-Aldrich; Merck KGaA, Darmstadt, Germany) was subcutaneously injected for six weeks. After one hour, 0 and $40 \mathrm{mg} / \mathrm{kg} / \mathrm{d} \mathrm{Sal} \mathrm{B} \mathrm{(Fig.} \mathrm{1)} \mathrm{was}$ injected into the model and Sal B groups, respectively, for three weeks.

Tissue sample preparation and hematoxylin and eosin (HE) staining. Rats were anesthetized with trichloroacetaldehyde hydrate $(0.3 \mathrm{ml} / \mathrm{kg}$; Sinopharm Chemical Reagent Co., Ltd, China) and subsequently sacrificed by exsanguination via an aortectomy. Left side tissue samples of bilateral femora were fixed for 3 days in $4 \%$ paraformaldehyde. Tissue samples were washed and dehydrated using gradient alcohol, and were subsequently embedded using paraffin wax. Tissue samples were cut into $4-\mu \mathrm{m}$ sections. These sections were stained using HE, and the slices were examined using a fluorescence microscope (Carl Zeiss, Oberkochen, Germany).

Evaluation of steroid-induced ONFH. Changes in the excised femoral head sample and bone trabeculae were detected using micro-CT ( $\mu \mathrm{CT}$; GE Healthcare Biosciences, Piscataway, NJ, USA). Bone volume (BV), trabecular bone pattern factor (Tb.Pf), trabecular thickness (Tb.Th), trabecular number (Tb.N), trabecular separation (Tb.Sp), and bone mineral density (BMD) were calculated (14).

Osteocalcin (OC) and alkaline phosphatase (ALP) content. Left side tissue samples of bilateral femora were acquired and homogenized using $100 \mu \mathrm{l}$ ice-cold lysis buffer $(10 \mathrm{mM}$ EGTA, $20 \mathrm{mM}$ Tris- $\mathrm{HCl}, 0.4 \% \mathrm{NaF}$, and protease inhibitors, 2 mM EDTA, pH 7.5) for 15 min. Subsequently, homogenates were centrifuged at $12,300 \times \mathrm{g}$ for $10 \mathrm{~min}$ at $4^{\circ} \mathrm{C}$ and a bicinchoninic acid protein assay kits (Beyotime Institute of Biotechnology, Nanjing, China) was used to determine the protein concentration. Equal protein $(10 \mu \mathrm{g})$ was used to measure OC and ALP using H152 and A059-2 kits (Nanjing Jiancheng Bioengineering Institute, Nanjing, China).

Cells and culture system. Bone marrow cells were obtained from the long bones of male C57 mice (age, 4-6 weeks old) obtained from the Experiment Center of Hebei Medical University. The cells were cultured in the presence of M-CSF (HyClone; GE Healthcare Life Sciences, Logan, UT, USA) for three days to separate the bone marrow-derived macrophages. Bone marrow-derived macrophages were cultivated with $40 \mu \mathrm{M} \mathrm{Sal} \mathrm{B}$ for four days. The presence of osteogenesis of the bone marrow-derived macrophages was determined by

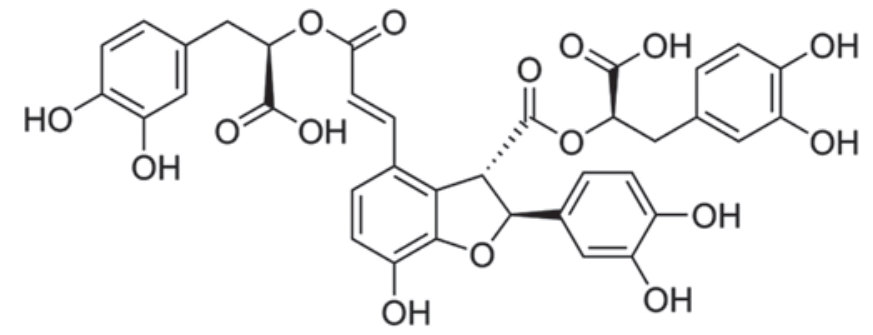

Figure 1. Chemical structure of salvianolic acid B.

Oil Red O staining, and positive cells were observed under a fluorescence microscope (Carl Zeiss).

Western blot analysis. Left side tissue samples of bilateral femora were acquired and homogenized using $100 \mu 1$ ice-cold lysis buffer (10 mM EGTA, $20 \mathrm{mM}$ Tris- $\mathrm{HCl}, 0.4 \% \mathrm{NaF}$, and protease inhibitors, $2 \mathrm{mM}$ EDTA, $\mathrm{pH}$ 7.5) for $15 \mathrm{~min}$. Subsequently, homogenates were centrifuged at $12,300 \times \mathrm{g}$ for $10 \mathrm{~min}$ at $4^{\circ} \mathrm{C}$ and a Bicinchoninic Acid protein assay (Beyotime Institute of Biotechnology, Nanjing, China) was used to determine the protein concentration. Next, 30-80 $\mu \mathrm{g}$ protein was separated using $12 \%$ sodium dodecyl sulfate-polyacrylamide gels and transferred onto polyvinylidene fluoride membranes. Blocking was performed with 5\% skim milk powder in TBST for $1 \mathrm{~h}$ at $37^{\circ} \mathrm{C}$. The following rabbit antibodies were used: PPAR $\gamma$ (1:300; sc-7273), AP2 (1:200; sc-12635), runtrelated transcription factor (Runx)2 (1:400; sc-101145), Collagen (Col) I (1:400; sc-59772) and GAPDH (1:2,000; all Santa Cruz Biotechnology, Inc., Dallas, TX, USA) overnight at $4^{\circ} \mathrm{C}$. The membrane was extensively washed using TBST for $1 \mathrm{~h}$ and probed with horseradish peroxidase-conjugated secondary antibody (1:5,000; sc-2005 or sc-2768; Santa Cruz Biotechnology, Inc.) for $2 \mathrm{~h}$. Relative band intensity was determined by a gel image analysis system (Bio-Rad Laboratories, Inc., Hercules, CA, USA).

Statistical analysis. SPSS version 13.0 software (SPSS Inc., Chicago, IL, USA) was used for statistical analysis. Numerical data are presented as the mean \pm standard deviation. Comparisons between two groups were performed using independent-sample t-test. $\mathrm{P}<0.05$ was considered to indicate a statistically significant difference.

\section{Results}

Sal B prevents histopathological changes in rats with steroid-induced osteonecrosis. The effect of Sal B on histopathological changes was examined in rats by steroid-induced osteonecrosis. As shown in Fig. 2, histopathological changes of control rats were notably reduced, as compared with the steroid-induced osteonecrosis model group. Histopathological changes of rats induced by steroid-induced osteonecrosis treatment with Sal B were markedly inhibited as compared with model group.

Sal $B$ prevents osteoclast differentiation in rats with steroid-induced osteonecrosis. Whether Sal B prevents osteoclast differentiation of bone marrow derived macrophages was 


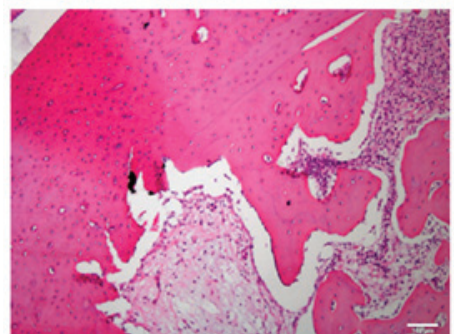

Sham

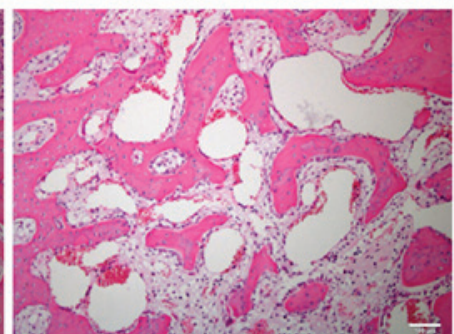

Model

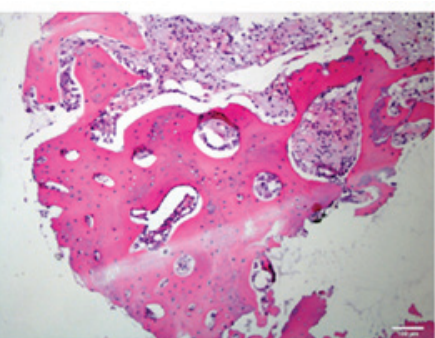

Sal B

Figure 2. Sal B prevents histopathological changes in rats with steroid-induced osteonecrosis (magnification, x100). Sham, control group; Model, steroid-induced osteonecrosis model group; Sal B, salvianolic acid B-treated group.
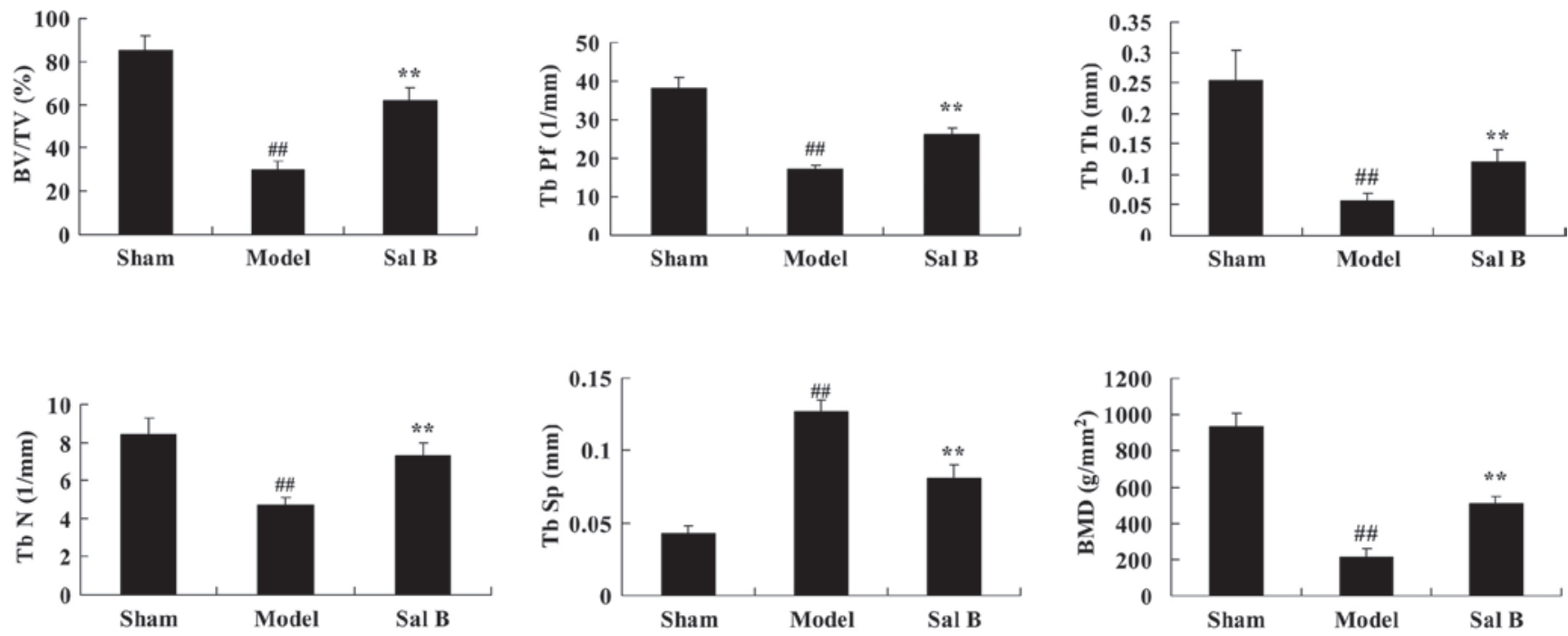

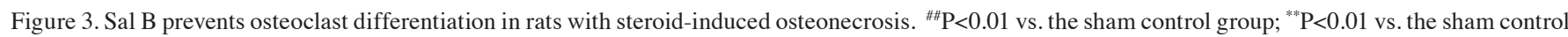
group. Sham, control group; Model, steroid-induced osteonecrosis model group; Sal B, salvianolic acid B-treated group; BV/TV, bone volume/total volume; Tb.Pf, trabecular bone pattern factor; Tb.Th, trabecular thickness; Tb.N, trabecular number; Tb.Sp, trabecular separation; BMD, bone mineral density.

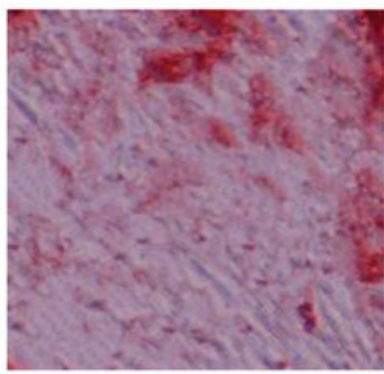

Control

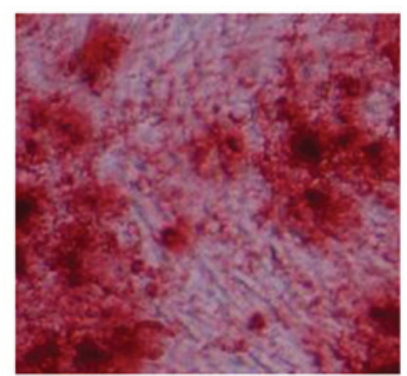

Sal B
Figure 4. Sal B accelerates osteogenesis in rats with steroid-induced osteonecrosis (magnification, $\mathrm{x} 100$ ). Sal B, salvianolic acid B-treated group.

investigated. As shown in Fig. 3, BV, Tb.Pf, Tb.Th, Tb.N and BMD values of the steroid model group were lower than that of the control group. Tb.Sp values of the steroid model group were higher than that the control group. However, treatment with Sal B significantly reversed the steroid-induced changes in BV, Tb.Pf, Tb.Th, Tb.N, Tb.Sp and BMD in rats $(\mathrm{P}<0.01)$.

Sal $B$ accelerates osteogenesis of bone marrow-derived macrophages. Following investigation of the effects of Sal B on the osteoclast differentiation of bone marrow-derived macrophages, Sal B was found to promote osteoclast differentiation of bone marrow-derived macrophages, as shown in Fig. 4.

Sal B prevents PPAR $\gamma$ signaling in rats with steroid-induced osteonecrosis. To determine the underlying mechanism associated with the effects of Sal B on steroid-induced osteonecrosis, the expression level of PPAR $\gamma$ protein was assessed by western blot analysis. As shown in Fig. 5, the expression levels of PPAR $\gamma$ protein were significantly increased by steroid treatment, as compared with the control group $(\mathrm{P}<0.01)$. Notably, pretreatment with Sal B significantly suppressed steroid-induced increases in the expression levels of PPAR $\gamma$ protein $(\mathrm{P}<0.01)$.

Sal B prevents AP2 signaling in rats with steroid-induced osteonecrosis. The endothelial protective effects of Sal B on AP2 signaling pathway were examined in rats with steroid-induced osteonecrosis using western blotting. As shown in Fig. 6, the AP2 protein expression levels in the control group were significantly lower than that of the steroid-induced osteonecrosis model group $(\mathrm{P}<0.01)$. In contrast, treatment with Sal B was demonstrated to significantly reduce steroid-induced AP2 signaling in rats via steroid-induced osteonecrosis $(\mathrm{P}<0.01)$. 

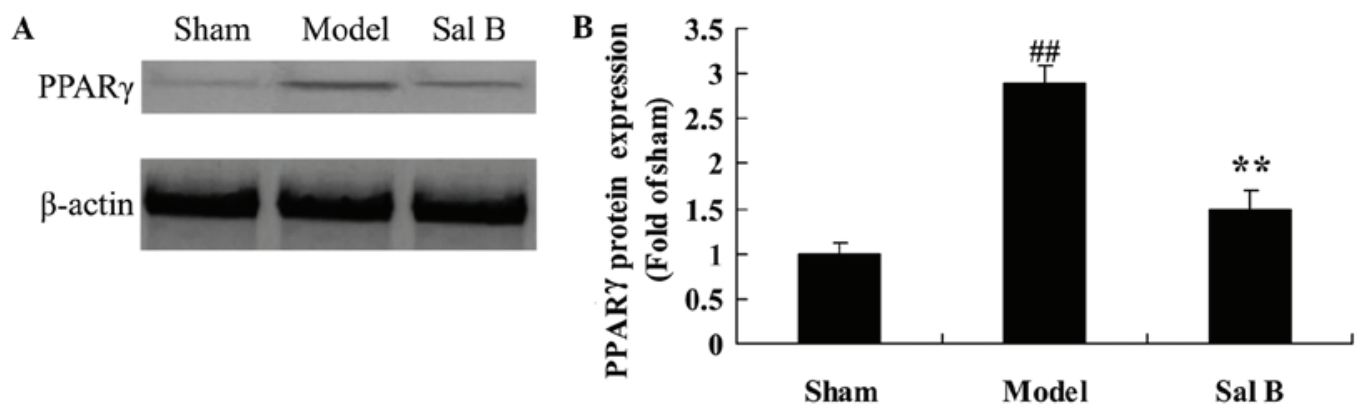

Figure 5. Salvianolic acid B prevents PPAR $\gamma$ signaling in rats with steroid-induced osteonecrosis. The effect of salvianolic acid B on PPAR $\gamma$ protein expression was investigated (A) using western blotting and (B) statistical analysis of PPAR $\gamma$ protein expression in rats by steroid-induced osteonecrosis. ${ }^{\# \#} \mathrm{P}<0.01 \mathrm{vs}$. the sham control group; ${ }^{* *} \mathrm{P}<0.01$ vs. the sham control group. Sham, control group; Model, steroid-induced osteonecrosis model group; Sal B, salvianolic acid B-treated group; PPAR, peroxisome proliferator-activated receptor.
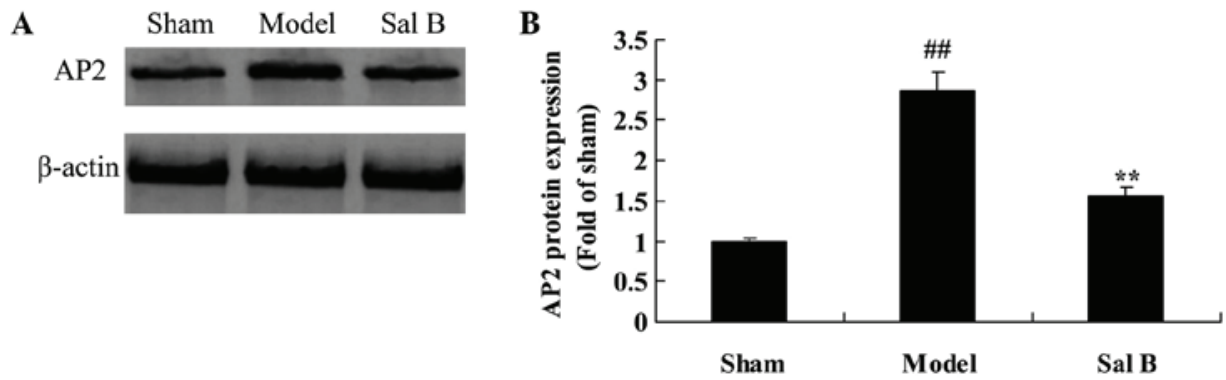

Figure 6. Sal B prevents AP2 signaling in rats with steroid-induced osteonecrosis. The effect of salvianolic acid B on AP2 protein expression was investigated (A) using western blotting and (B) statistical analysis of AP2 protein expression in rats by steroid-induced osteonecrosis. ${ }^{\# /} \mathrm{P}<0.01$ vs. the sham control group; ${ }^{* *} \mathrm{P}<0.01$ vs. the sham control group. Sham, control group; Model, steroid-induced osteonecrosis model group; Sal B, salvianolic acid B-treated group.
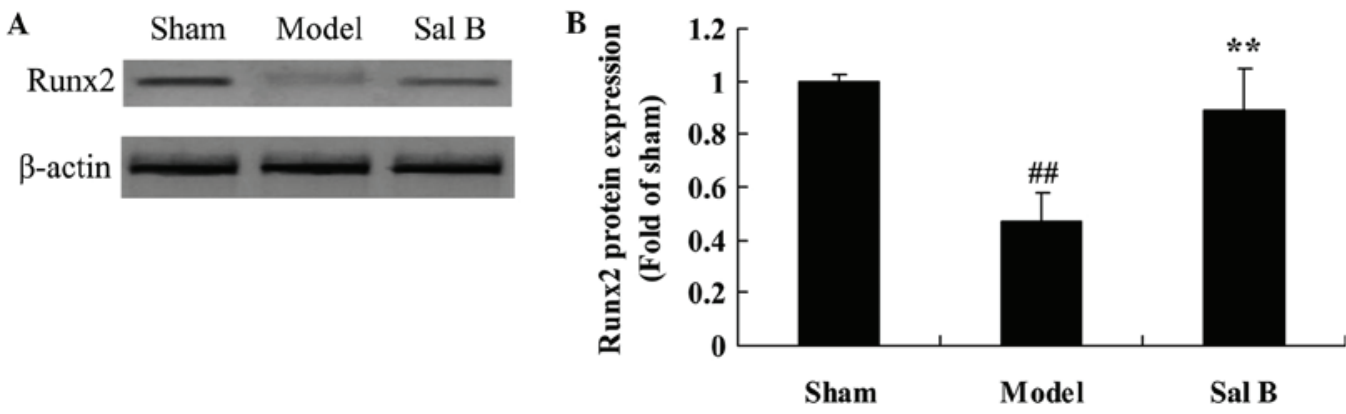

Figure 7. Sal B increases Runx2 signaling in rats with steroid-induced osteonecrosis. The effect of salvianolic acid B on Runx2 protein expression was investigated (A) using western blotting and (B) statistical analysis of Runx2 protein expression in rats by steroid-induced osteonecrosis. ${ }^{\# \#<0.01 ~ v s . ~ t h e ~ s h a m ~}$ control group; ${ }^{* *} \mathrm{P}<0.01$ vs. the sham control group. Sham, control group; Model, steroid-induced osteonecrosis model group; Sal B, salvianolic acid B-treated group; Runx2, runt-related transcription factor (Runx)2.

Sal B increases Runx2 signaling in rats with steroid-induced osteonecrosis. The endothelial protective effects of Sal B on Runx 2 protein expression in rats were quantified by steroid-induced osteonecrosis. As shown in Fig. 7, there was an significantly decrease in Runx 2 protein expression of steroid-induced osteonecrosis, as compared with the control group $(\mathrm{P}<0.01)$. Pretreatment with Sal B significantly increased Runx 2 protein expression levels of steroid-induced osteonecrosis rats $(\mathrm{P}<0.01)$.

Sal B accelerates Col I signaling in rats with steroid-induced osteonecrosis. In order to determine whether the effect of Sal B accelerates Col I signaling pathway in rats with steroid-induced osteonecrosis, Col I protein expression was measured using western blot analysis. As shown in Fig. 8, significant steroid-induced inhibition of Col I signaling was observed in the model group, as compared with control group $(\mathrm{P}<0.01)$. However, following treatment with Sal B, steroid-induced Col I signaling was significantly increased in rat with steroid-induced osteonecrosis $(\mathrm{P}<0.01)$.

Sal $B$ increases $O C$ and $A L P$ content in rats with steroid-induced osteonecrosis. To assess the effect of Sal B on osteonecrosis, OC and ALP content in rats with steroid-induced osteonecrosis were evaluated. As shown in Fig. 9, OC and ALP contents were significantly lower than those of control 
A

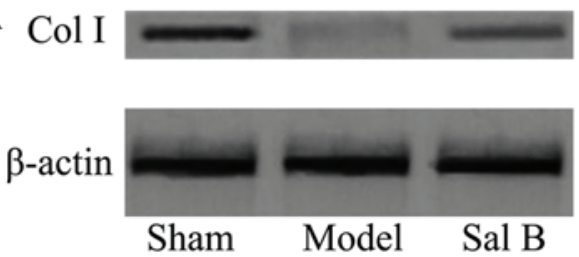

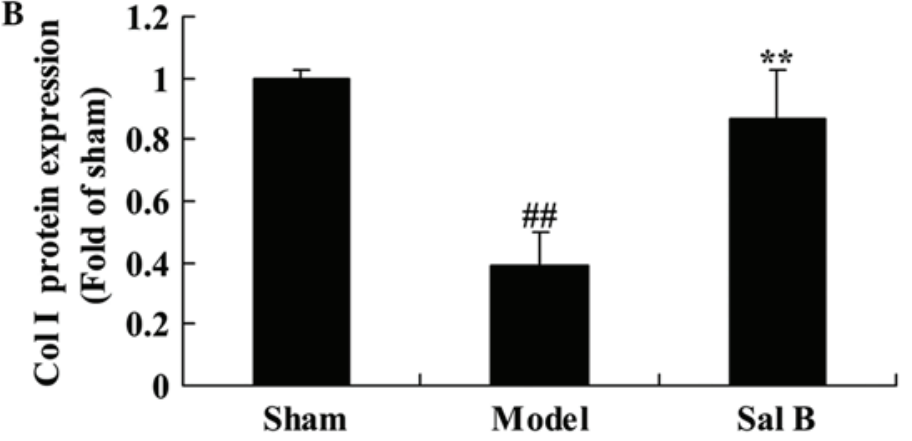

Figure 8. Sal B increases Col I signaling in rats with steroid-induced osteonecrosis. The effect of salvianolic acid B on Col I protein expression was investigated (A) using western blotting and (B) statistical analysis of Col I protein expression in rats by steroid-induced osteonecrosis. ${ }^{\# \#} \mathrm{P}<0.01$ vs. the sham control group; ${ }^{* *} \mathrm{P}<0.01$ vs. the sham control group. Sham, control group; Model, steroid-induced osteonecrosis model group; Sal B, salvianolic acid B-treated group; Col, collagen.
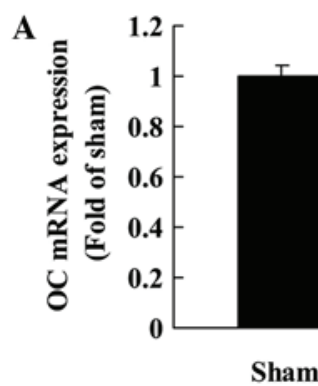
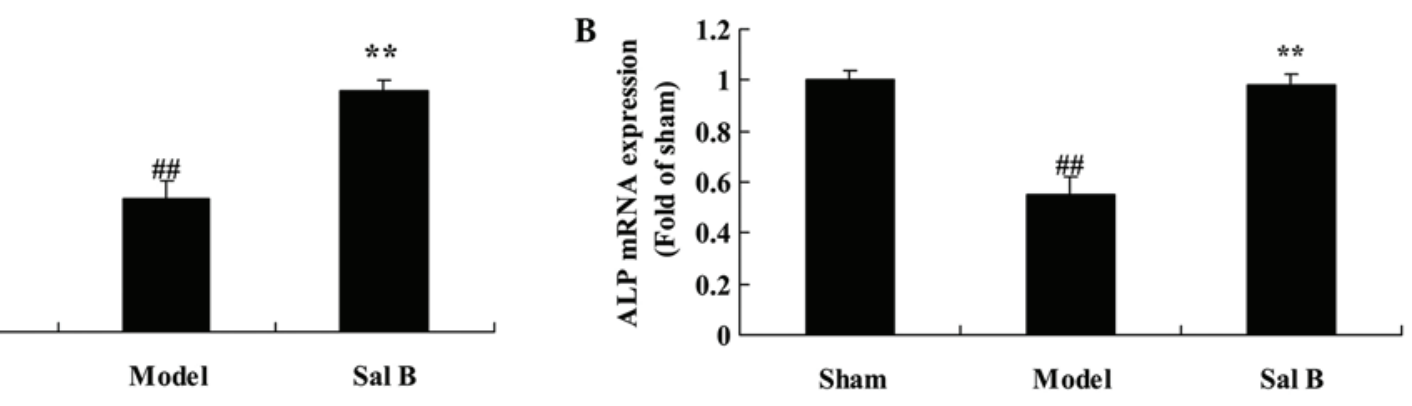

Figure 9. Sal B increases (A) OC and (B) ALP content in rats with steroid-induced osteonecrosis. ${ }^{\# \#} \mathrm{P}<0.01$ vs. the sham control group; ${ }^{* *} \mathrm{P}<0.01$ vs. the sham control group. Sham, control group; Model, steroid-induced osteonecrosis model group; Sal B, salvianolic acid B-treated group; OC, osteocalcin; ALP, alkaline phosphatase.

group $(\mathrm{P}<0.05)$. However, Sal B significantly increased steroid-induced the inhibition of OC and ALP contents in rats with steroid-induced osteonecrosis $(\mathrm{P}<0.01)$.

\section{Discussion}

In recent years, hormone imbalance has become the most prevalent reason for non-traumatic osteonecrosis, with the widespread clinical application of hormonal drugs (2). There are various treatments for the disease; however, the effects are not optimal. The main aim of treatment is to reduce the progressive destruction of joint cartilage and subchondral bone and to prevent the collapse of the femoral head (3). As for the collapsed femoral head, surgical methods are predominantly used for treatment, including total hip joint replacement; although these surgical techniques may have a therapeutic effect, surgical complications and economic burden to the patients should not be disregarded (7). Therefore, it is critical that a safe, efficient, economical and non-invasive prevention method is found. The results of the present study demonstrated that Sal B prevents histopathological changes and osteoclast differentiation in rats with steroid-induced osteonecrosis, and accelerates the osteogenesis of bone marrow derived macrophages. These findings indicate that Sal B may protect against steroid-induced osteonecrosis. Cui et al (15) reported that Sal B prevents bone loss through the stimulation of osteogenesis and bone marrow angiogenesis by PPAR $\gamma$ mRNA expression and Runx 2 mRNA expression in prednisone-treated rats.
Xu et al (16) confirmed that Sal B promotes the osteogenesis of human mesenchymal stem cells via osteopontin, Runx 2 and osterix expression.

Hormones are able to activate enhancer binding protein (C/EBPS), thereby activating the expression of PPAR- $\gamma$, and inducing the differentiation of bone marrow stromal stem cells into adipocytes (17). It has been proposed that large doses of corticosteroids may induce bone marrow stromal stem cells to upregulate the expression of PPAR- $\gamma$ mRNA in cell culture and genetic studies (18). Since bone marrow stromal stem cells can be differentiated into both bone cells and fat cells, adipocyte differentiation is increased, whereas osteoblast differentiation is reduced accordingly (19). In vitro experimentation has demonstrated that hormones can induce the MSCs cultured in vitro to elevate the expression of PPAR- $\gamma$ by as much as 2 -fold, so that the core binding factor a1 (Cbfa1/Runx2) reduces by $50-60 \%$, inducing differentiation into adipocytes and reducing osteoblast differentiation (20). Studies have shown that, under the optimal conditions, adipocytes and osteoblasts may be converted into each other, since the femoral head in osteoblasts and adipocytes share a common pool of bone marrow stromal cells $(21,22)$. Thus the generation of a large number of fat cells induced by hormones is an important mechanism of femoral head necrosis disease (23). In the present study, pretreatment with Sal B significantly suppressed steroid-induced expression levels of PPAR $\gamma$ and AP2 protein.

Runx 2 and Osterix are considered to be the most important transcription factors that regulate the stereotype and differ- 
entiation of osteoblasts, which are the key site for a variety of hormones that regulate the osteoblast differentiation and bone formation of local and external regulatory factors (24). Scholars have confirmed that high-concentration hormones are able to inhibit the expression and activity of osteoblast phenotype markers Runx 2 and Osterix by investigating in-vitro cell culture and the molecular and genetic level (25). In present study, pretreatment with Sal B significantly increased Runx 2 and Col I protein expression levels in steroid-induced osteonecrosis rats. This indicates the potential role of PPAR $\gamma / \mathrm{AP} 2 / \mathrm{Runx} 2 / \mathrm{Col} \mathrm{I}$ in the Sal B-induced effects on steroid-induced osteonecrosis. Wang et al (26) suggested that Sal B inhibited PPAR $\gamma$ expression in mice with high-fat diet-induced obesity. Cui et al (15) reported that Sal B prevents bone loss through the stimulation of osteogenesis and bone marrow angiogenesis via PPAR $\gamma$ and Runx 2 mRNA expression in prednisone-treated rats.

Mesenchymal stem cells are directionally induced into osteoblasts in osteogenic medium. The contents of ALP and BGP are determined after PPAR- $\gamma$ cells are lysed (27). ALP is capable of hydrolyzing organophosphate, so that the local concentration of the phosphate is increased, which may damage calcification inhibitor to initiate calcification (7). Therefore, improving the ALP content is an important indicator for the differentiation of mesenchymal stem cells into osteoblasts, and the level of ALP content in the cells may be used to measure the presence and differentiation degree of osteoblasts (28). In the present study, Sal B significantly increased the steroid-induced inhibition of OC and ALP content in rats with steroid-induced osteonecrosis.

In conclusion, the present study showed that Sal B prevents SANFH via PPAR $\gamma$ expression in rats. In characterizing the mechanism of action, we discovered that the PPAR $\gamma / \mathrm{AP} 2 / \mathrm{Run} \times 2 / \mathrm{Col} \mathrm{I}$ and OC/ALP pathways are required for both Sal B-mediated protective effects against steroid-induced osteonecrosis. Therefore, Sal B should be considered a potential osteonecrosis drug for the treatment of steroid-induced osteonecrosis.

\section{References}

1. Fan SH, Wang YY, Lu J, Zheng YL, Wu DM, Zhang ZF, Shan Q, Hu B, Li MQ and Cheng W: CERS2 suppresses tumor cell invasion and is associated with decreased V-ATPase and MMP-2/MMP-9 activities in breast cancer. J Cell Biochem 116: $502-513,2015$.

2. Xie M, Hu A, Luo Y, Sun W, Hu X and Tang S: Interleukin-4 and melatonin ameliorate high glucose and interleukin-1 $\beta$ stimulated inflammatory reaction in human retinal endothelial cells and retinal pigment epithelial cells. Mol Vis 20: 921-928, 2014.

3. Adams BD, Kasinski AL and Slack FJ: Aberrant regulation and function of microRNAs in cancer. Curr Biol 24: R762-R776, 2014.

4. Tang C, Chen L, Gu W, Du M, Li M, Chen Q and Li D: Cyclosporin A enhances the ability of trophoblasts to displace the activated human umbilical vein endothelial cell monolayers. Int J Clin Exp Pathol 6: 2441-2450, 2013.

5. Maxwell GL, Shoji Y, Darcy K, Litzi T, Berchuck A, Hamilton CA, Conrads TP and Risinger JI: MicroRNAs in endometrial cancers from black and white patients. Am J Obstet Gynecol 212: 191. e1-e10, 2015.

6. Seven M, Karatas OF, Duz MB and Ozen M: The role of miRNAs in cancer: From pathogenesis to therapeutic implications. Future Oncol 10: 1027-1048, 2014.

7. Saracoglu I and Harput US: In vitro cytotoxic activity and structure activity relationships of iridoid glucosides derived from Veronica species. Phytother Res 26: 148-152, 2012.
8. Cao W, Guo XW, Zheng HZ, Li DP, Jia GB and Wang J: Current progress of research on pharmacologic actions of salvianolic acid B. Chin J Integr Med 18: 316-320, 2012.

9. Tian J, Fu F, Li G, Gao Y, Zhang Y, Meng Q, Li C and Liu F: Protections of SMND-309, a novel derivate of salvianolic acid B, on brain mitochondria contribute to injury amelioration in cerebral ischemia rats. Phytomedicine 16: 726-733, 2009.

10. Yang TL, Lin FY, Chen YH, Chiu JJ, Shiao MS, Tsai CS, Lin SJ and Chen YL: Salvianolic acid B inhibits low-density lipoprotein oxidation and neointimal hyperplasia in endothelium-denuded hypercholesterolaemic rabbits. J Sci Food Agric 91: 134-141, 2011.

11. Durairajan SS, Yuan Q, Xie L, Chan WS, Kum WF, Koo I, Liu C, Song Y, Huang JD, Klein WL and Li M: Salvianolic acid B inhibits Abeta fibril formation and disaggregates preformed fibrils and protects against Abeta-induced cytotoxicty. Neurochem Int 52: 741-750, 2008.

12. Tang MK and Zhang JT: Salvianolic acid B inhibits fibril formation and neurotoxicity of amyloid beta-protein in vitro. Acta Pharmacol Sin 22: 380-384, 2001.

13. Pan CS, Liu YH, Liu YY, Zhang Y, He K, Yang XY, Hu BH, Chang X, Wang MX, Wei XH, et al: Salvianolic Acid B ameliorates lipopolysaccharide-induced albumin leakage from rat mesenteric venules through Src-regulated transcelluar pathway and paracellular pathway. PLoS One 10: e0126640, 2015.

14. Wang L, Zhang L, Pan H, Peng S, Zhao X and Lu WW: Abnormal subchondral bone microstructure following steroid administration is involved in the early pathogenesis of steroid-induced osteonecrosis. Osteoporos Int 27: 153-159, 2016.

15. Cui L, Li T, Liu Y, Zhou L, Li P, Xu B, Huang L, Chen Y, Liu Y, Tian X, et al: Salvianolic acid B prevents bone loss in prednisone-treated rats through stimulation of osteogenesis and bone marrow angiogenesis. PLoS One 7: e34647, 2012.

16. Xu D, Xu L, Zhou C, Lee WY, Wu T, Cui L and Li G: Salvianolic acid $\mathrm{B}$ promotes osteogenesis of human mesenchymal stem cells through activating ERK signaling pathway. Int J Biochem Cell Biol 51: 1-9, 2014.

17. Xiao M, Wang $X$ and Chen W: The clinical translational potential of p53-related alterations as cancer biomarkers. Histol Histopathol 30: 1171-1183, 2015.

18. Yi J and Luo J: SIRT1 and p53, effect on cancer, senescence and beyond. Biochim Biophys Acta 1804: 1684-1689, 2010.

19. Hoban DB, Howard L and Dowd E: GDNF-secreting mesenchymal stem cells provide localised neuroprotection in an inflammation-driven rat model of Parkinson's disease. Neuroscience 303: 402-411, 2015.

20. Emamghoreishi M, Keshavarz M and Nekooeian AA: Acute and chronic effects of lithium on BDNF and GDNF mRNA and protein levels in rat primary neuronal, astroglial and neuroastroglia cultures. Iran J Basic Med Sci 18: 240-246, 2015.

21. Herzog DP, Dohle E, Bischoff I and Kirkpatrick CJ: Cell communication in a coculture system consisting of outgrowth endothelial cells and primary osteoblasts. Biomed Res Int 2014: 320123, 2014.

22. Dumont N, Boyer L, Emond H, Celebi-Saltik B, Pasha R, Bazin R, Mantovani D5, Roy DC and Pineault N: Medium conditioned with mesenchymal stromal cell-derived osteoblasts improves the expansion and engraftment properties of cord blood progenitors. Exp Hematol 42: 741-752 e741, 2014.

23. Bender TS, Migliore MM, Campbell RB, John Gatley S and Waszczak BL: Intranasal administration of glial-derived neurotrophic factor (GDNF) rapidly and significantly increases whole-brain GDNF level in rats. Neuroscience 303: 569-576, 2015.

24. Pogribny IP, Filkowski JN, Tryndyak VP, Golubov A, Shpyleva SI and Kovalchuk O: Alterations of microRNAs and their targets are associated with acquired resistance of MCF-7 breast cancer cells to cisplatin. Int J Cancer 127: 1785-1794, 2010.

25. Nunes de Carvalho S, Helal-Neto E, de Andrade DC, Costa Cortez EA, Thole AA, Barja-Fidalgo $\mathrm{C}$ and de Carvalho L: Bone marrow mononuclear cell transplantation increases metalloproteinase- 9 and 13 and decreases tissue inhibitors of metalloproteinase- 1 and 2 expression in the liver of cholestatic rats. Cells Tissues Organs 198: 139-148, 2013.

26. Wang P, Xu S, Li W, Wang F, Yang Z, Jiang L, Wang Q, Huang M and Zhou P: Salvianolic acid B inhibited PPAR $\gamma$ expression and attenuated weight gain in mice with high-fat diet-induced obesity. Cell Physiol Biochem 34: 288-298, 2014.

27. Liu YF, Zhao Y, Wen XS and Dong QT: Advances in research on pharmacodynamics and chemical conversion of catalpol. Zhongguo Zhong Yao Za Zhi 32: 1128-1130, 2007 (In Chinese).

28. Yarbro $\mathrm{CH}$ : International nursing and breast cancer. Breast $\mathbf{J} 9$ (Suppl 2): S98-S100, 2003. 